\title{
Markov states of the quantum electromagnetic field
}

\author{
Luigi Accardi and Geoffrey S. Watson \\ Princeton University, Fine Hall, Washington Road, Princeton, New Jersey 08544
}

(Received 11 August 1986)

\begin{abstract}
A translation-invariant state (a quantum Markov chain) is associated with a nearest-neighbor interaction on a one-dimensional lattice by a new technique which provides closed forms for all the correlation functions. When applied to an Ising-type perturbation of a chain of harmonic oscillators, the dynamics can be computed explicitly. The resulting translation-invariant distribution is substantially different from the Planck distribution when the temperature and the coupling constant are large. For the evolution of the field operators on a given mode, we obtain a natural nonlinear generalization of the theorem which states that the free evolution of the field operators is obtained by second quantization of the classical free evolution.
\end{abstract}

\section{INTRODUCTION}

In the present paper we use techniques of quantum Markov chains to associate a translation-invariant state with a large class of nearest-neighbor interactions on a one-dimensional lattice in such a way that the correlation functions to any order can be computed in closed form. In view of the general applicability of the method, we recall, in Sec. II, some basic facts of the theory of quantum Markov chains (cf. Ref. 1 for a review). At the end of Sec. II this technique is compared with the usual one, based on a cutoff and limiting-taking procedure. In Sec. III we apply the above technique to an Ising-type perturbation of a chain of harmonic oscillators-an example in which all the computations can be made explicitly. Other examples will be discussed in subsequent papers. The remaining sections are devoted to the computation of the main physical parameters associated with this interaction (mean and variance of the number operator and of the linear functions of the field and time as well as mode correlations and spectra) and to the comparison of the results with those obtained when $\lambda$ (i.e., the interaction) is equal to zero in which case our formal (i.e., nonrenormalized) Hamiltonian reduces to the usual Hamiltonian of the free Gibbs state. This comparison is particularly instructive in the case of the invariant distribution of the number of photons in a given mode as a function of $\lambda$ which is not exponential like the Planck distribution (to which it reduces when the interaction parameter $\lambda$ is set equal to 0 ). Some numerical computations (cf. Table I below) of this distribution show that the discrepancy of the probability of the number states from the values of the Planck distribution at the same temperature can be quite remarkable, i.e., of the order of $20 \%$. Hence it should be possible to distinguish experimentally between the states with $\lambda \neq 0$ and the free Gibbs state. Another interesting fact is that while the invariant equilibrium distribution is not of the Planck type, the conditional distribution of $N_{j}$ given $N_{j-1}$ is Planck type, but at a lower temperature than the one of the corresponding free state. This new temperature depends on both the interaction parameter $\lambda$ and the number of photons in the conditioning mode. We call this effect the "cooling effect of the interaction" (cf. Sec. IV).

\section{THE STATE ASSOCIATED WITH A NEAREST-NEIGHBOR INTERACTION}

Consider a one-dimensional one-sided lattice whose points are identified with the natural integers $N=0,1,2, \ldots$. [when an invariant density operator exists, the construction can be extended to the case of a twosided lattice-cf. the remarks following formula (2.14) below]. To each point $j$ of the lattice we associate the Hilbert space $\mathscr{H}_{j}$ of the $j$ th mode of the field.

On each of the spaces $\mathscr{H}_{j}$ we have a representation of the boson commutation relations with creation and annihilation operators denoted $a_{j}^{\dagger}, a_{j}$, respectively. We assume that the representation $a, a^{\dagger}$ is irreducible so that all the spaces $\mathscr{H}_{j}$ are isomorphic to a single Hilbert space $H_{0}$. In the following, for any Hilbert space $\mathscr{K}$ we will denote $\mathscr{B}(\mathscr{K})$ the algebra of all bounded operators on $\mathscr{K}$.

Objects related to the space $\mathscr{H}_{j}$ will be labeled with the subscript index $j$ : i.e., $\operatorname{Tr}_{j}$ denotes the trace of operators on $\mathscr{H}_{j} ; A_{j}$ is a generic observable of the $j$ th mode of the field, e.g., $N_{j}=a_{j}^{\dagger} a_{j}$ is the number of photons in the $j$ th mode, $q_{j}=(1 / \sqrt{2})\left(a_{j}+a_{j}^{\dagger}\right)$ the electric field of the $j$ th mode, $p_{j}=(i / \sqrt{2})\left(a_{j}-a_{j}^{\dagger}\right)$ the magnetic field of the $j$ th mode, etc.; $\left|n_{j}\right\rangle$ is the $n_{j}$ th state of the operator $N_{j}$. If $m \leq n$, the Hilbert space associated with the modes from $m$ to $n$ will be denoted $\mathscr{H}_{[m, n]}$. Thus

$$
\mathscr{H}_{[m, n]}=\mathscr{H}_{m} \otimes \mathscr{H}_{m+1} \otimes \cdots \otimes \mathscr{H}_{n}
$$

If an observable depends only on the modes in the interval $[m, n]$ then we say that it is localized on $[m, n]$ (e.g., $q_{[m, n]}=[1 /(n-m+1)] \sum_{j=m}^{n} q_{j}$ is the average electric field over the modes in $[m, n])$. Sometimes, by analogy with the multidimensional case, we refer to the interval $[m, n]$ as the "volume."

An infinite-volume state, i.e., a state of the electromagnetic (em) field, is defined by a sequence $W_{[0, n]}$ of density operators localized on finite intervals and such that the limit 


$$
\lim _{n \rightarrow \infty} \operatorname{Tr}_{[0, n]}\left(W_{[0, n]} X\right)=\varphi(X)
$$

exists for any observable $X$ localized on any finite interval. By density arguments this limit will then exist for any observable.

We want to associate a state of the em field to a given nearest-neighbor interaction. By definition a nearestneighbor interaction is a family of self-adjoint operators $H(n, n+1)$ localized on the interval $[n, n+1]$. The free Hamiltonian of the $n$th mode will be denoted $H_{n}$ and we write

$$
Z_{\beta}(n)=\operatorname{Tr}\left(e^{-\beta H_{n}}\right)
$$

for the partition function. In the transition-invariant case we simply write $Z_{\beta}$. The theory of quantum Markov chains is a technique to associate with a large class of nearest-neighbor interactions $H(n, n+1)$, an infinitevolume state according to the following prescriptions: one first defines the renormalized conditional density amplitude

$$
K_{n}=e^{-H_{n+1} / 2} e^{-H(n, n+1) / 2} e^{-H_{n} / 2} S_{n}^{-1 / 2},
$$

where $S_{n}$ is the operator defined by

$$
\begin{aligned}
S_{n}=\operatorname{Tr}_{n+1}^{\prime}\left(e^{-H_{n}} e^{-H(n, n+1) / 2}\right. & \\
& \left.\times e^{-H_{n+1}} e^{-H(n, n+1) / 2} e^{-H_{n}}\right)
\end{aligned}
$$

and $\operatorname{Tr}_{n+1}^{\prime}$ denotes the partial trace over the space $\mathscr{H}_{n+1}$ [i.e., if $A_{1}, \ldots, A_{n+1}$ are operators localized on the modes $1, \ldots, n+1$, respectively, then $\left.\operatorname{Tr}_{n+1}^{\prime}\left(A_{1} \ldots A_{n} A_{n+1}\right)=A_{1} \ldots A_{n} \operatorname{Tr}_{n+1}\left(A_{n+1}\right)\right]$. [Our method is applicable when the partial trace $(2.4)$ produces a well-defined invertible operator.]

The two key properties of the operators $K_{n}$ defined by (2.3) are

$$
\begin{aligned}
& K_{n} \text { is localized on }[n, n+1], \\
& \operatorname{Tr}_{n+1}^{\prime}\left(K_{n}^{*} K_{n}\right)=1,
\end{aligned}
$$

where the right-hand side denotes the identity operator on the space $\mathscr{H}_{n}$. In the construction that follows only these two properties of the operators $K_{n}$ will be used [and not the explicit form (2.3)]. In the theory of quantum Markov chains an operator enjoying the two properties (2.5) and (2.6) is called a conditional density amplitude: as the von Neumann density matrix is the quantum analogue of the usual probability density, the conditional density amplitude is the quantum analogue of the transition (or conditional) probability density (or better, of its square root, in the same way as a wave function is a square root of a probability density).

Now choose an arbitrary density operator $w_{0}$ localized on the zero mode and define, for each $n$, the operator

$$
W_{[0, n]}=K_{n} K_{n-1} \cdots K_{0} w_{0} K_{0}^{*} K_{1}^{*} \cdots K_{n}^{*} \text {. }
$$

Using (2.5) and (2.6) it is easy to verify that each $W_{[0, n]}$ is a density operator. Notice that $W_{[0, n]}$ is localized on $[0, n+1]$. Furthermore, condition (2.6) above assures that, for each observable $A_{[0, m]}$ localized on $[0, m]$ ( $m$ an arbitrary natural integer) the limit

$$
\varphi\left(A_{[0, m]}\right)=\lim _{n \rightarrow \infty} \operatorname{Tr}_{[0, n]}\left(W_{[0, n]} A_{[0, m]}\right)
$$

exists and is equal to

$$
\operatorname{Tr}_{[0, m+1]}\left(W_{[0, m]} A_{[0, m]}\right)
$$

In fact, since $K_{n}$ is localized on $[n, n+1]$ and $A_{[0, m]}$ in $[0, m], A_{[0, m]}$ and $K_{n}$ commute for $m<n$ so one finds

$$
\begin{aligned}
\operatorname{Tr}_{[0, n+1]}\left(W_{[0, n]} A_{[0, m]}\right) \\
\quad=\operatorname{Tr}_{[0, n+1]}\left(K_{n} \cdots K_{0} w_{0} K_{0}^{*} \cdots K_{n}^{*} A_{[0, m]}\right) \\
\quad=\operatorname{Tr}_{[0, n+1]}\left(K_{n-1} \cdots K_{0} w_{0} K_{0}^{*} \cdots K_{n-1}^{*} A_{[0, m]} K_{n}^{*} K_{n}\right) \\
\quad=\operatorname{Tr}_{[0, n+1]}\left[K_{n-1} \cdots K_{0} w_{0} K_{0}^{*} \cdots K_{n-1}^{*} A_{[0, m]} \operatorname{Tr}_{n+1}^{\prime}\left(K_{n}^{*} K_{n}\right)\right]=\operatorname{Tr}_{[0, n]}\left(K_{n-1} \cdots K_{0} w_{0} K_{0}^{*} \cdots K_{n-1}^{*} A_{[0, m]}\right)
\end{aligned}
$$

Hence, by iterating the procedure we arrive at (2.9) where we must stop since it is no longer true that $K_{m} A_{[0, m]}$ $=A_{[0, m]} K_{m}$.

In many interesting examples the Hamiltonian is translation invariant, i.e., $H_{n}$ and $H(n, n+1)$ are obtained by translating by $n$ steps $H_{0}$ and $H(0,1)$, respectively. In these cases one would like the state $\varphi$, defined by $(2.8)$, to be translation invariant; that is, denoting by $\sigma$ the operator which shifts the observables one step to the right [e.g., $\sigma\left(N_{j}\right)=N_{j+1}$, we want the $\varphi$-expected value of any observable $A$ to be equal to the $\varphi$-expected value of the shifted observable $\sigma(A)$. A simple calculation shows that $\varphi$ is translation invariant if and only if the mode zero dis- tribution $w_{0}$ satisfies

$$
\operatorname{Tr}_{0}^{\prime}\left(K_{0} w_{0} K_{0}^{*}\right)=\sigma\left(w_{0}\right) \text {. }
$$

For a general translation-invariant interaction $H(0,1)$, it might not be easy to solve equation (2.11). However, if, as happens in several interesting examples, the interaction $H(0,1)$ is symmetric in the two modes, i.e., it is invariant under the flip automorphism $\delta$, which exchanges the observables of the two modes $[\delta(A \otimes B)=B \otimes A ; A, B$ observables], then one can actually construct a solution of (2.11) (which in many cases turns out to be the unique solution). In fact, considering the operator $S_{0}$, defined by Eq. (2.4) with $n=0$, and using the definition (2.3) of $K_{n}$, we obtain 
$\operatorname{Tr}_{0}^{\prime}\left(K_{0} S_{0} K_{0}^{*}\right)$

$$
=\operatorname{Tr}_{0}^{\prime}\left(e^{-H_{1} / 2} e^{-H(0,1) / 2} e^{-H_{0} / 2} e^{-H(0,1) / 2} e^{-H_{1} / 2}\right) .
$$

Now, by assumption $H(0,1)$ is symmetric, hence $\delta\left(\exp \left[-\frac{1}{2} H(0,1)\right]\right)=\exp \left[-\frac{1}{2} H(0,1)\right] ;$ moreover, the translation invariance of the free Hamiltonian implies that $\delta\left(H_{0}\right)=H_{1}$ and $\delta\left(H_{1}\right)=H_{0}$. Since clearly $\operatorname{Tr}_{0}^{\prime} \circ \delta$ $=\sigma \circ \operatorname{Tr}_{1}^{\prime}$, it follows that the right-hand side of $(2.12)$ is equal to

$$
\begin{aligned}
\operatorname{Tr}_{0}^{\prime} \circ \delta\left(e^{-H_{0} / 2} e^{-H(0,1) / 2} e^{-H_{1}} e^{-H(0,1) / 2} e^{-H_{0} / 2}\right) \\
\quad=\sigma \circ \operatorname{Tr}_{1}^{\prime}\left(e^{-H_{0} / 2} e^{-H(0,1) / 2} e^{-H_{1}} e^{-H(0,1) / 2} e^{-H_{0} / 2}\right) \\
\quad=\sigma\left(S_{0}\right)
\end{aligned}
$$

where in the last equality we have used the definition (2.4) of $S_{0}$. Thus, if $S_{0}$ is trace class, we can define

$$
w_{0}=S_{0} / \operatorname{Tr}\left(S_{0}\right)
$$

Otherwise we take simply $w_{0}=S_{0}$ and we obtain what in the theory of classical Markov chains is called an infinite invariant measure.

Summing up: to every single-mode Hamiltonian $H_{0}$ and symmetric nearest-neighbor-interaction Hamiltonian $H(0,1)$ whose associated [by Eq. (2.4) with $n=0$ ] operator $S_{0}$ is invertible, we can associate in a very explicit and constructive way a translation-invariant state of the em field on a one-dimensional lattice.

Notice that, in the translation-invariant case, the construction above can be extended to the two-sided onedimensional lattice simply defining the expectation of an observable localized on $[-m, n]$ as the expectation of the same observable shifted $m$ steps to the right.

A particularly simple situation is obtained when everything in the above construction commutes, i.e., when in addition to flip invariance the operators $H_{0}$ and $H(0,1)$ satisfy

$$
\begin{aligned}
& {\left[H_{0}, H(0,1)\right]=0,} \\
& {[H(0,1), H(1,2)]=0 .}
\end{aligned}
$$

Then one has also

$$
\left[H(0,1), S_{0}\right]=\left[H_{0}, S_{0}\right]=0
$$

and because of translation invariance, all these relations remain true when one substitutes $n$ and $n+1$ for 0 and 1 , respectively. Moreover, the sequence $W_{[0, n]}$ of density operators associated to the translation-invariant Hamiltonian defined by the pair $\left\{H_{0}, H(0,1)\right\}$ takes the particularly simple form

$$
\begin{aligned}
W_{[0, n]} & =w_{0} P_{0} P_{1} \cdots P_{n-1} \\
& =\left[\prod_{j=0}^{n-1} S_{n}^{-1}\right] \exp \left[-\sum_{j=0}^{n} H_{j}-\sum_{j=0}^{n-1} H(j, j+1)\right],
\end{aligned}
$$

where $W_{0}$ is given by (2.14) and

$$
P_{n}=K_{n}^{*} K_{n}=S_{n}^{-1} e^{-\left[H_{n}+H(n, n+1)+H_{n+1}\right]}, n \geq 0
$$

(by convention $\prod_{j=1}^{n-1}=1$ and $\sum_{j=1}^{n-1}=0$ if $n=0$ ). Notice that, contrarily to the general situation in which $W_{[0, n]}$ is localized on $[0, n+1]$, in this commuting situation $W_{[0, n]}$ is localized on $[0, n]$. This happens because, when we perform the limit (2.8) under the commutation conditions (2.15), (2.16), and (2.17), we obtain, in the notations of (2.19) and (2.18),

$\varphi\left(A_{[0, m+1]}\right)=\operatorname{Tr}_{[0, m+1]}\left(w_{0} P_{0} P_{1} \cdots P_{m-1} P_{m} A_{[0, m]}\right)$

and since $P_{m}$ commutes with $P_{j}$ for $j \leq m$ as well as with $w_{0}$, this is equal to

$$
\begin{aligned}
& \operatorname{Tr}_{[0, m+1]}\left(P_{m} w_{0} P_{0} P_{1} \cdots P_{m-1} A_{[0, m]}\right) \\
& \quad=\operatorname{Tr}_{[0, m]}\left[\operatorname{Tr}_{m+1}^{\prime}\left(P_{m}\right) w_{0} P_{0} \cdots P_{m-1} A_{[0, m]}\right] \\
& \quad=\operatorname{Tr}_{[0, m]}\left(w_{0} P_{0} \cdots P_{m-1} A_{[0, m]}\right)
\end{aligned}
$$

and this proves (2.18).

The entropy density $S=S\left(H_{0}, H(0,1)\right)$ of a state defined by the sequence $(2.18)$ of density operators has a simple expression given by

$$
\begin{aligned}
S & =-\lim _{n \rightarrow \infty} \frac{1}{n+1} \operatorname{Tr}_{[0, n]}\left(W_{[0, n]} \ln W_{[0, n]}\right) \\
& =-\lim _{n \rightarrow \infty} \frac{1}{n+1}\left[\operatorname{Tr}_{[0, n]}\left(W_{[0, n]} \ln w_{0}\right)+\sum_{j=0}^{n} \operatorname{Tr}_{[0, n]}\left(W_{[0, n]} \ln P_{j}\right)\right] \\
& =-\lim _{n \rightarrow \infty} \frac{1}{n+1}\left[\operatorname{Tr}_{0}\left(w_{0} \ln w_{0}\right)+n \operatorname{Tr}_{[0,1]}\left(w_{0} P_{0} \ln P_{0}\right)\right]=-\operatorname{Tr}_{[0,1]}\left(w_{0} P_{0} \ln P_{0}\right)
\end{aligned}
$$


and using the expressions (2.14) for $w_{0}$ [here we are assuming $\left.\operatorname{Tr}_{0}\left(S_{0}\right)<\infty\right]$ and (2.19) for $P_{0}$ we eventually obtain

$$
\begin{aligned}
S= & -\lim _{n \rightarrow \infty} \frac{1}{n+1} \operatorname{Tr}_{[0, n]}\left(W_{[0, n]} \ln W_{[0, n]}\right) \\
= & \frac{\operatorname{Tr}_{0}\left(S_{0} \ln S_{0}\right)}{\operatorname{Tr}_{0}\left(S_{0}\right)}+2 \frac{\operatorname{Tr}_{0}\left(S_{0} H_{0}\right)}{\operatorname{Tr}\left(S_{0}\right)} \\
& +\frac{\operatorname{Tr}_{[0,1]}\left[e^{-\left[H_{0}+H(0,1)+H_{1}\right]} H(0,1)\right]}{\operatorname{Tr}\left(S_{0}\right)} .
\end{aligned}
$$

Also the Heisenberg dynamics of such a state is easily calculated. For any observable $A_{[0, m]}$ localized on $[0, m]$ one has

$$
\begin{aligned}
\lim _{n \rightarrow \infty} & W_{[0, n]}^{-i t} A_{[0, m]} W_{[0, n]}^{i t} \\
& =w_{0}^{-i t} P_{0}^{-i t} \cdots P_{m}^{-i t} A_{[0, m]} P_{m}^{i t} \cdots P_{0}^{i t} w_{0}^{i t}=u_{t}\left(A_{[0, m]}\right) .
\end{aligned}
$$

From this it follows immediately that, if in our construction we rescale the Hamiltonians by $H_{n} \rightarrow \beta H_{n}$, $H(n, n+1) \rightarrow \beta H(n, n+1)$, then the resulting state satisfies the Kubo-Martin-Schwinger condition at inverse temperature $\beta$. In particular, if $A_{k}$ is an observable localized on the site $k$ of the lattice, then if $k \geq 1$

$$
u_{t}\left(A_{k}\right)=P_{k-1}^{i t} P_{k}^{i t} P_{k}^{i t} A_{k} P_{k}^{-i t} P_{k-1}^{-i t}
$$

while if $k=0$

$$
u_{t}\left(A_{0}\right)=w_{0}^{i t} P_{0}^{i t} A_{0} P_{0}^{-i t} w_{0}^{-i t} .
$$

In the following sections we discuss in detail the infinite-volume state associated with the procedure described above for a particular nearest-neighbor interaction satisfying the commutativity conditions (2.15) and (2.16). Notice that for interactions of this type one can easily define a (nonrenormalized) dynamics on the local observables as the limit

$$
\lim _{M \rightarrow \infty} \exp \left[-i t\left(\sum_{j=0}^{M}\left[H_{j}+H(j, j+1)\right]\right)\right] A \text {. }
$$

But, in order to associate a state with this dynamics, one should prove that there exist a positive operator $B_{0}$ and a strictly positive number $t$ such that [in the notations introduced before formula (2.11)]

$$
\operatorname{Tr}_{2}^{\prime}\left[e^{-\beta / 2\left[H_{0}+\lambda H(0,1)\right]} \sigma\left(B_{0}\right) e^{-\beta / 2\left[H_{0}+H(0,1)\right]}\right]=t B_{0}
$$

Furthermore one should prove that the number $t$ is a simple eigenvalue for the operator on the left hand side of (2.27) and that it is the only one whose modulus is $t$. This fact would assure the existence of the limit

$$
\lim _{M \rightarrow \infty} \frac{\operatorname{Tr}\left[\exp \left[-\beta \sum_{j=1}^{M}\left[H_{j}+H(0,1)\right]\right] A\right]}{\operatorname{Tr}\left[\exp \left[-\beta \sum_{j=0}^{n}\left[H_{j}+H(0,1)\right]\right]\right.}
$$

for any local observable $A$. Once the solution of Eq. (2.27) is available it is not difficult to obtain a translation-invariant state. However, when the state space $\mathscr{H}_{0}$ is infinite dimensional, one knows very little about the existence of solutions of (2.27) with the required properties, and even less about their explicit form. The renormalization (2.3) plus the symmetry trick (2.12) and (2.13) allow us to bypass this problem and to obtain in an easy way an invariant state.

\section{THE $N_{j} N_{j+1}$ INTERACTION}

We consider a one-dimensional one-sided infinite chain of harmonic oscillators in which the force exerted on the $j$ th oscillator is not only proportional to its displacement but also to the energy of its nearest neighbors. Denoting by $H_{j}=\frac{1}{2}\left(q_{j}^{2}+p_{j}^{2}\right)$ the Hamiltonian of the $j$ th oscillator, the equations of motion for such an oscillator are then

$$
\begin{aligned}
& \dot{q}_{j}=p_{j}\left[\lambda H_{j-1}+(1-\lambda)+\lambda H_{j+1}\right], \\
& \dot{p}_{j}=-q_{j}\left[\lambda H_{j-1}+(1-\lambda)+\lambda H_{j+1}\right]
\end{aligned}
$$

for $j \geq 1$ and, since the zero mode has only one neighbor,

$$
\begin{aligned}
& \dot{q}_{0}=p_{0}\left[(1-\lambda / 2)+\lambda H_{1}\right], \\
& \dot{p}_{0}=-q_{0}\left[(1-\lambda / 2)+\lambda H_{1}\right] .
\end{aligned}
$$

In the above equations, and in this paper, $\lambda$ denotes a coupling constant. The formal Hamiltonian of such a system is

$$
H_{\text {class }}=(1-\lambda / 2) H_{0}+(1-\lambda) \sum_{j=1}^{\infty} H_{j}+\lambda \sum_{j=0}^{\infty} H_{j} H_{j+1} .
$$

This system is completely integrable as a classical system: in it the $H_{j}$ can be taken as action variables and the phases correspond to the complex representation

$$
q_{j}+i p_{j}=z_{j}=H_{j} e^{i \phi_{j}} \text {. }
$$

Now let us (boson) quantize this system. In terms of creation and annihilation operators, the formal Hamiltonian becomes

$$
H_{\text {quant }}=\sum_{j=0}^{\infty} N_{j}+\lambda \sum_{j=0}^{\infty} N_{j} N_{j+1},
$$

where $N_{j}$ is the number operator relative to the $j$ th mode. This interaction appears for Fermi oscillators, and in a Hamiltonian including also other interacting terms, in some papers by Fedyanin, Makhankov, and Yakushevich ${ }^{4}$ devoted to the analysis of possible soliton behavior in the exciton-phonon interaction in the long-wavelength approximation. Also, at the quantum level one could easily obtain a limiting dynamics from the finite-volume approximations of the Hamiltonian. However, the existence of a limiting translation-invariant state requires the solution of an operator eigenvalue problem which, even if feasible in this case, would be in general quite impracticable.

Now we apply to the interaction described above the method of Sec. II. In the notations of the preceding section, we now choose the free Hamiltonian to be the number operator (in appropriate units), 


$$
H_{j}=N_{j},
$$

and the interaction Hamiltonian to be the one of the nonlinear chain of harmonic oscillators discussed in the Introduction, i.e., of the form

$$
H(j, j+1)=\lambda N_{j} N_{j+1}
$$

so that the associated Hamiltonian is both translation invariant and flip invariant. In the following we identify the space $H_{0} \otimes H_{0}$ with any of the spaces $\mathscr{H}_{j} \otimes \mathscr{H}_{j}$ and the suffix $j$ appended to an operator acting on $H_{0}$ means that we are letting this operator act on $\mathscr{H}_{j}$. With these nota- tions we introduce, for any positive real number $\beta$, denoting the inverse temperature, the unnormalized conditional amplitude

$$
\begin{aligned}
\exp \left[\frac{-\beta}{2}(N \otimes 1+\lambda N \otimes N+1 \otimes N)\right] \\
\simeq e^{-(\beta / 2)\left(N_{j}+\lambda N_{j} N_{j+1}+N_{j+1}\right)}
\end{aligned}
$$

with $\lambda \geq 0$, which is clearly flip invariant. Thus, recalling the notation (2.4),

$$
\begin{aligned}
S_{0}=\operatorname{Tr}_{2}^{\prime}\left(K_{u}^{*} K_{u}\right) & =\operatorname{Tr}_{1}^{\prime}\left(K_{u}^{*} K_{u}\right) \\
& =\operatorname{Tr}_{2}^{\prime}\left(e^{-\beta N \otimes 1-\beta \lambda N \otimes N-\beta 1 \otimes N}\right)=\operatorname{Tr}_{2}^{\prime}\left(\sum_{m, n} e^{-\beta m-\beta \lambda m n-\beta n}|m\rangle\langle m|\otimes| n\rangle\langle n|\right) \\
& =\sum_{m} \frac{e^{-\beta m}}{1-e^{-\beta(\lambda m+1)}}|m\rangle\langle m|=f_{\lambda, \beta}(N),
\end{aligned}
$$

where we have introduced the notation

$$
f_{\lambda, \beta}(n)=\frac{e^{-\beta n}}{1-e^{-\beta(\lambda n+1)}} .
$$

Therefore, following the prescriptions of Sec. II, we can define the normalized conditional density amplitude

$$
\begin{aligned}
K & =K_{u}\left[f_{\lambda, \beta}(N) \otimes 1\right]^{-1 / 2} \\
& =\left(1-e^{-\beta(\lambda N \otimes 1+1)}\right)^{1 / 2}\left(e^{-(\lambda \beta / 2) N \otimes N-(\beta / 2) 1 \otimes N}\right)
\end{aligned}
$$

and the density matrix

$$
w=f_{\beta, \lambda}(N) / Z_{\beta, \lambda}=\frac{1}{Z_{\beta, \lambda}} e^{-\beta N}\left(1-e^{-\beta(\lambda N+1)}\right)^{-1},
$$

where the partition function $Z_{\beta, \lambda}$ is defined by

$$
\begin{aligned}
Z_{\beta, \lambda} & =\operatorname{Tr}\left(S_{0}\right) \\
& =\operatorname{Tr}\left[f_{\lambda, \beta}(N)\right] \\
& =\sum_{m} \frac{e^{-\beta m}}{1-e^{-\beta(\lambda m+1)}} \leq \frac{1}{\left(1-e^{-\beta}\right)^{2}} .
\end{aligned}
$$

It is not difficult to see that, as a function of $\lambda$, the partition function $Z_{\beta, \lambda}$ is an analytic function in the halfplane $\operatorname{Re} z>0$ while in the negative half-plane it has poles in the points

$$
\lambda=\frac{1}{m}+\frac{2 \pi k i}{m \beta}
$$

( $k$ and $m$ integer) and it is still analytic in the strips delimited by two vertical lines drawn over two consecutive poles. This behavior of $Z_{\beta, \lambda}$ suggests that one might obtain a more interesting phase diagram than the one ob- tained for $\lambda>0$ if one could extend our method to a region of negative $\lambda$.

The pair $w, K$ will define, according to the discussion of Sec. II, a stationary Markov chain $\varphi$ on the algebra $\mathscr{A}=\bigotimes_{j \in N} \mathscr{B}\left(\mathscr{H}_{j}\right)=\bigotimes_{N} \mathscr{B}\left(H_{0}\right)$ with the property that for any observable a localized on a finite interval one has

$$
\varphi(a)=\operatorname{Tr}_{[0, n]}\left(W_{[0, n]} a\right), a \in \mathscr{A}_{[0, n]}
$$

with $W_{[0, n]}$ given by

$$
\begin{aligned}
W_{[0, n]} & =K_{n-1} K_{n-2} \cdots K_{0} w_{0} K_{0}^{*} K_{1}^{*} \cdots K_{n-1}^{*} \\
& =w_{0} P_{0} \cdots P_{n-1},
\end{aligned}
$$

where $w_{0}$ denotes $w$ acting on $\mathscr{H}_{0}$ and the transition operator $P$ is defined, according to the prescription (2.19), by

$$
P=K^{*} K=\left(1-e^{-\beta(\lambda N \otimes 1+1)}\right) e^{-\lambda \beta N \otimes N-\beta 1 \otimes N} .
$$

Thus the transition probability between the $j$ th and the $(j+1)$ th mode takes the particularly simple form

$$
\begin{aligned}
P_{j}=j_{j}(P) & =\left(1-e^{-\beta\left(\lambda N_{j}+1\right)}\right) e^{-\lambda \beta N_{j} N_{j+1}-\beta N_{j+1}} \\
& =\sum_{m_{j}=0}^{\infty}\left|m_{j}\right\rangle\left\langle m_{j}\right| \otimes \frac{e^{-\beta\left(\lambda m_{j}+1\right) N_{j+1}}}{Z\left(\beta\left(\lambda m_{j}+1\right)\right)},
\end{aligned}
$$

where the index $j$ in $m_{j}$ means that the state is referred to the $j$ th mode and $Z(\beta(\lambda m+1))$ is defined by

$$
Z(m)=Z(\beta(\lambda m+1))=\left(1-e^{-\beta(\lambda m+1)}\right)^{-1},
$$


i.e., it is the usual Planck factor at inverse temperature $(\lambda m+1) \beta$. Using the explicit expression for $w_{0}$ and $P_{j}$, for every $n$, the density matrix of the restriction of $\varphi$ on $\mathscr{A}_{[0, n]}$ can be written explicitly:

$$
\begin{aligned}
W_{[0, n]}= & \frac{1}{Z_{\lambda, \beta}} \exp \left[-\beta \sum_{j=0}^{n} N_{j}-\beta \lambda \sum_{j=0}^{n-1} N_{j} N_{j+1}\right] \\
& \times\left[\prod_{j=1}^{n-1}\left(1-e^{-\beta\left(\lambda N_{j}+1\right)}\right)\right],
\end{aligned}
$$

where by definition, if $n=1$ the product is equal to 1 . In particular

$$
W_{[0,1]}=\frac{1}{Z_{\beta, \lambda}} e^{-\beta\left(N_{0}+N_{1}+\lambda N_{0} N_{1}\right)} .
$$

Notice that both the density operator $w$, defined by (3.7), and the transition operator $P$ have a direct physical meaning. In fact, the former defines the Hamiltonian

$$
H_{\lambda, \beta}=-\ln f_{\lambda, \beta}(N)+\ln Z_{\beta, \lambda},
$$

which is the renormalized Hamiltonian of the single modes and defines the invariant distribution of the chain; and the operator $\boldsymbol{P}$ can be naturally interpreted as "transition density operator" defined by a "renormalized interac- tion Hamiltonian." The analogy with the classical Markov chains is evident and in the following section this analogy will be made more precise.

\section{THE NUMBER-OPERATOR PROCESS}

The family of random variables $\left(N_{j}\right)$ corresponding to the occupation numbers of all the modes of the field with the distribution induced by the state $\varphi$ will be called the number-operator process. From the explicit formula (3.14) of the sequence of density matrices $W_{[0, n]}$ it follows that $\left(N_{j}\right)$ is a classical stationary Markov process with transition matrix

$$
\begin{aligned}
P\left(N_{j+1}=n \mid N_{j}\right. & =m) \\
& =p_{m, n}=e^{-\beta(\lambda m+1) n}\left(1-e^{-\beta(\lambda m+1)}\right)
\end{aligned}
$$

and invariant initial distribution

$$
p_{n}^{0}(\lambda)=p_{n}^{0}=\frac{1}{Z_{\beta, \lambda}} \frac{e^{-\beta n}}{1-e^{-\beta(\lambda n+1)}}=f_{\lambda, \beta}(n) / Z_{\lambda, \beta} .
$$

\begin{tabular}{|c|c|c|c|c|c|}
\hline & & $\beta=0.1$ & $\beta=0.5$ & $\beta=1$ & $\beta=5$ \\
\hline \multirow[t]{4}{*}{$\lambda=0$} & $Z$ & 110.42 & 6.459 & 2.503 & 1.014 \\
\hline & $p_{0}$ & 0.0952 & 0.393 & 0.632 & 0.993 \\
\hline & $p_{1}$ & 0.0861 & 0.238 & 0.233 & 0.006 \\
\hline & $p_{2}$ & 0.0791 & 0.145 & 0.086 & 0.000 \\
\hline \multirow[t]{4}{*}{$\lambda=0.1$} & $Z$ & 70.225 & 5.900 & 2.433 & 1.014 \\
\hline & $p_{0}$ & 0.150 & 0.431 & 0.650 & 0.993 \\
\hline & $p_{1}$ & 0.124 & 0.243 & 0.227 & 0.007 \\
\hline & $p_{2}$ & 0.103 & 0.079 & 0.080 & \\
\hline \multirow[t]{4}{*}{$\lambda=0.5$} & $Z$ & 41.101 & 5.000 & 2.296 & 1.014 \\
\hline & $p_{0}$ & 0.256 & 0.508 & 0.689 & 0.993 \\
\hline & $p_{1}$ & 0.158 & 0.230 & 0.206 & 0.007 \\
\hline & $p_{2}$ & 0.110 & 0.116 & 0.068 & \\
\hline \multirow[t]{4}{*}{$\lambda=1$} & $Z$ & 32.101 & 4.595 & 2.230 & 1.014 \\
\hline & $p_{0}$ & 0.327 & 0.553 & 0.710 & 0.993 \\
\hline & $p_{1}$ & 0.155 & 0.209 & 0.191 & 0.007 \\
\hline & $p_{2}$ & 0.098 & 0.103 & 0.006 & \\
\hline \multirow[t]{4}{*}{$\lambda=5$} & $Z$ & 21.911 & 4.116 & 2.165 & 1.014 \\
\hline & $p_{0}$ & 0.480 & 0.617 & 0.731 & 0.993 \\
\hline & $p_{1}$ & 0.092 & 0.155 & 0.170 & 0.007 \\
\hline & $p_{2}$ & 0.056 & 0.090 & 0.006 & \\
\hline \multirow[t]{4}{*}{$\lambda=10$} & $z$ & 20.633 & 4.085 & 2.164 & 1.014 \\
\hline & $p_{0}$ & 0.509 & 0.622 & 0.731 & 0.993 \\
\hline & $p_{1}$ & 0.065 & 0.149 & 0.170 & 0.007 \\
\hline & $p_{2}$ & 0.005 & 0.009 & 0.006 & \\
\hline \multirow[t]{4}{*}{$\lambda=100$} & Z & 20.016 & 4.083 & 2.164 & \\
\hline & $p_{0}$ & 0.525 & 0.622 & 0.731 & \\
\hline & $p_{1}$ & 0.005 & 0.149 & 0.170 & \\
\hline & $p_{2}$ & 0.004 & 0.009 & 0.063 & \\
\hline
\end{tabular}

This is most easily seen by writing the density matrix $W_{[0, n]}$ in (3.14) in the number-operator representation, i.e.,

$$
W_{[0, k]}=\sum_{n_{0}, n_{1}, \ldots, n_{k}} p_{n_{0}}^{0} p_{n_{0}, n_{1}} \cdots p_{n_{k-1}, n_{k}}\left|n_{0}\right\rangle\left\langle n_{0}|\otimes| n_{1}\right\rangle\left\langle n_{1}|\otimes \cdots \otimes| n_{k}\right\rangle\left\langle n_{k}\right|,
$$

TABLE I. $Z_{\beta, \lambda}$ and photon distributions for various $\beta, \lambda . \lambda=0$ gives Planck distribution. 
where the mode zero distribution $p_{n}^{0}$ is given by (3.7), and the transition matrix $\left(p_{i, j}\right)$ is defined by $(4.1)$. Notice that for $\lambda=0$ one has

$$
Z_{\beta, 0}=\left(1-e^{-\beta}\right)^{2}
$$

while for $\lambda \rightarrow \infty$ one has

$$
Z_{\beta, \infty}=\operatorname{coth}(\beta / 2) \text {. }
$$

Moreover by explicit computation of the derivative $(\partial / \partial \lambda) Z_{\beta, \lambda}$ one sees that $Z_{\beta, \lambda}$ decreases monotonically from $Z_{\beta, 0}$ to $Z_{\beta, \infty}$. Correspondingly, for $\lambda=0$ in (4.2) we find the Planck distribution, while for $\lambda=\infty$ we find

$$
\begin{aligned}
& p_{0}^{0}(\infty)=\frac{1}{\operatorname{coth}(\beta / 2)\left(1-e^{-\beta}\right)}, \\
& p_{n}^{0}(\infty)=\frac{e^{-\beta n}}{\operatorname{coth}(\beta / 2)}, \quad n \geq 1 .
\end{aligned}
$$

Notice that $p_{n}^{0} \geq p_{n+1}^{0}$ for each $n$. The transition matrix (4.1) corresponds to an antiferromagnetic Ising interaction in which, however, the "spin" variables do not vary in a compact space, but over all the natural integers. In particular, for each $j$, the conditional distribution of the number of quanta in the mode $N_{j+1}$ conditioned by the presence of $m$ quanta in the mode $j$ is a Planck distribution at the lower temperature $T^{\prime}=[\beta(\lambda m+1)]^{-1}$ ("cooling" effect of the interaction). In Table I we have reported the values of $Z_{\beta, \lambda}$ and the values of the probabilities of the first three number states $(n=0,1,2)$ for various choices of $\beta$ and $\lambda$. In particular, as a function of $\lambda$, these values tend to stabilize after $\lambda=10$, while in the range $0<\lambda<10$, the discrepancy with Planck's law $(\lambda=0)$ is large enough to be detected experimentally.
One can now apply the known results on Markov chains with countable state space to the Markov chain defined by the transition probability (4.1). In particular, since $p_{m, n}>0$ for all $m$ and $n$, then the Markov chain is irreducible, aperiodic, positive recurrent, and mixing. This means in particular that if we call $T(n)$ the gap between two successive occurrence in two different modes of the number state $|n\rangle$ then the mean value of $T(n)$ will be equal to

$$
\frac{1}{p_{n}^{0}}=e^{\beta n}\left(1-e^{-\beta(\lambda n+1)}\right) Z_{\beta, \lambda} .
$$

Moreover, from the estimate

$$
p_{m, n} \leq \frac{Z_{\beta, \lambda}}{1-e^{-\beta}} p_{n}^{0}
$$

one easily deduces (via Ref. 5, p. 209) that there are constants $\gamma$ and $\rho$ with $0<\rho<1$ such that for all integer $m$ and for any set of integers $A$ one has

$$
\left|\sum_{n \in A}\left[P_{k}(m, n)-p_{n}^{0}\right]\right| \leq \gamma \rho^{k},
$$

where $P_{k}(m, n)$ denotes the $(m, n)$ coefficient of the $k$ th power of the matrix $P$. This implies an exponential decay of correlations (i.e., no thermodynamical phase transitions).

\section{THE ENTROPY}

From the explicit formula (3.14) of the density matrix we can compute the entropy density:

$$
\begin{aligned}
S\left(W_{[0, n]}\right) & =-\operatorname{Tr}_{[0, n]}\left(W_{[0, n]} \ln W_{[0, n]}\right) \\
& =\beta \sum_{j=0}^{n} \operatorname{Tr}_{j}\left(W_{j} N_{j}\right)+\lambda \beta \sum_{j=0}^{n-1} \operatorname{Tr}_{[j, j+1]}\left(W_{[j, j+1]} N_{j} N_{j+1}\right)-\sum_{j=1}^{n} \operatorname{Tr}_{j}\left[W_{j} \ln \left(1-e^{-\beta\left(\lambda N_{j}+1\right)}\right)\right] \\
& =-\operatorname{Tr}(w \ln w)+n \beta \operatorname{Tr}(w N)+\lambda \beta n \operatorname{Tr}_{[0,1]}\left(W_{[0,1]} N_{0} N_{1}\right)-n \operatorname{Tr}\left[w \ln \left(1-e^{-\beta(\lambda N+1)}\right)\right]
\end{aligned}
$$

hence the entropy density of $\varphi$ is

$$
\begin{aligned}
S(\varphi)= & \lim _{n \rightarrow \infty} \frac{1}{n} \operatorname{Tr}_{[0, n]}\left(W_{[0, n]} \ln W_{[0, n]}\right) \\
= & \beta \operatorname{Tr}(w N)+\lambda \beta \operatorname{Tr}_{[0,1]}\left(W_{[0,1]} \ln W_{[0,1]}\right) \\
& -\operatorname{Tr}\left[w \ln \left(1-e^{-\beta(\lambda N+1)}\right)\right] .
\end{aligned}
$$

The various terms on the right-hand side of (5.2) are easily computed, taking into account the expression (3.7) of $w$, and one finds

$$
\begin{aligned}
S(\varphi)= & -\frac{1}{Z_{\lambda, \beta}} \sum_{m=0}^{\infty} f_{\lambda, \beta}(m) \ln f_{\lambda, \beta}(m) \\
& +\frac{\lambda e^{-\beta}}{Z_{\lambda, \beta}} \sum_{m=0}^{\infty} m e^{\beta(1-\lambda) m} f_{\lambda, \beta}(m)^{2} .
\end{aligned}
$$

Up to the additive factor $Z_{\lambda, \beta}$ the first term in the expression (5.3) for the entropy density is easily seen to coincide with the entropy density of a free Gibbs state corresponding to the Hamiltonian (3.16).

\section{THE FIELD PROCESSES}

We want now to calculate the joint probability distribution at different times and relative to different modes of observables which are linear combinations of the electric and magnetic fields. First notice that, for $j \geq 1$, the evolution of the field observables $A_{j}=u^{*} a_{j}+u a_{j}^{\dagger}$ is easily computed using (2.24) and one finds

$$
A_{1}(u, t)=A_{1}\left(e^{i t \beta\left[\left(\lambda N_{0}+1\right)-q_{\beta, \lambda}\left(N_{1}\right)-\lambda N_{2}\right]} u\right)
$$


Notice that, up to the renormalization factor $q_{\beta, \lambda}\left(N_{1}\right)$, which vanishes for $\lambda=0$, this is exactly the secondquantized form of the solution of the classical equations of motion. This result suggests the conjecture that a similar theorem might hold for all the completely integrable classical systems. Towards this goal, we begin with a single-field observable $A(u)=u^{*} a+u a^{\dagger}$ at time zero, relative to the zero mode (for simplicity, we drop, for the moment, the index zero). In order to evaluate the characteristic function (Wigner distribution) of this observable in the given state we begin by remarking that for any number state $|m\rangle$ one has

$$
\begin{aligned}
\hat{p}_{A(u t)}(t \mid m)=\left\langle m, e^{i t A(u)} m\right\rangle & =e^{-t^{2}|u|^{2} / 2} \sum_{k=0}^{m}(-1)^{k} \frac{\left(t^{2}|u|^{2}\right)^{k}}{(k !)^{2}} \frac{m !}{(m-k) !} \\
& =e^{t^{2}|u|^{2} / 2} \frac{1}{m !} \sum_{l=o}^{m}\left(-t^{2}|u|^{2}\right)^{m-1}\left[\frac{m !}{(m-l) !}\right]^{2}=e^{-t^{2}|u|^{2} / 2} L_{m}\left(t^{2}|u|^{2}\right)
\end{aligned}
$$

where $L_{m}(x)$ denotes the $m$ th Laguerre polynomial normalized so that they form an orthonormal system for the measure $e^{-x} d x$ and $L_{m}(0)=1$ [cf. Ref. 2, p. 93, where, however, the normalization $L_{m}(0)=m$ ! is used]. In particular, since the characteristic function of the observable $A(u)$ in the state $|m\rangle$ is integrable, this distribution admits a probability density $p_{A(u)}(x \mid m)$ with respect to the Lebesgue measure given by

$$
P_{A(u)}(x \mid m)=\frac{1}{2 \pi} \int_{+\infty}^{-\infty} e^{-i x t} e^{-t^{2}|u|^{2} / 2} L_{m}\left(t^{2}|u|^{2}\right) d t=\frac{1}{\pi} \int_{0}^{\infty} \cos (x t) e^{-t^{2}|u|^{2} / 2} L_{m}\left(t^{2}|u|^{2}\right) d t
$$

The right-hand side of (6.2) is evaluated using the formula (cf. Ref. 4, p. 846, 7.4182)

$$
m ! \int_{0}^{\infty} e^{-y^{2} / 2} \cos (b y) L_{m}\left(y^{2}\right) d y=\left(\frac{\pi}{2}\right]^{1 / 2} e^{-b^{2} / 2} 2^{-m}\left[H_{m}(b / \sqrt{2})\right]^{2}
$$

where $b \in R$ and $H_{m}(x)$ denotes the $m$ th Hermite polynomial normalized so that

$$
\int_{-\infty}^{+\infty} e^{-x^{2}} H_{n}(x) H_{m}(x) d x=\delta_{m, n} 2^{m} m ! \sqrt{\pi} .
$$

Using (6.3) with $b=x /|u|$ and $y=t|u|$, we obtain

$$
p_{A(u)}(x \mid m)=\frac{1}{|u|} \frac{1}{\sqrt{2 \pi}} e^{-x^{2} / 2|u|^{2}} \frac{H_{m}^{2}(x / \sqrt{2}|u|)}{m ! 2^{m}} .
$$

From this the characteristic function of $A(u)$ in the state $\varphi$ is easily obtained. In fact, by translation invariance,

$$
\begin{aligned}
\varphi\left(e^{i t A(u)}\right)=\operatorname{Tr}\left(w_{0} e^{i t A(u)}\right) & =\frac{1}{Z_{\beta, \lambda}} \sum_{m=0}^{\infty} \frac{e^{-\beta m}}{1-e^{-\beta(\lambda m+1)}}\left\langle m\left|e^{i t A(u)}\right| m\right\rangle \\
& =\frac{1}{Z_{\beta, \lambda}} \sum_{m=0}^{\infty} \frac{e^{-\beta m}}{e^{-\beta(\lambda m+1)} e^{t^{2}\left|u^{2}\right| / 2} L_{m}\left(t^{2}|u|^{2}\right) .}
\end{aligned}
$$

Therefore

$$
p_{A(u)}(x \mid \varphi)=\frac{1}{Z_{\beta, \lambda}} \sum_{m=0}^{\infty} \frac{e^{-\beta m}}{1-e^{-\beta(\lambda m+1)}} P_{A(u)}(x \mid m)=\frac{1}{Z_{\beta, \lambda}} \sum_{m=0}^{\infty} \frac{e^{-\beta m}}{1-e^{-\beta(\lambda m+1)}} h_{m}(x ;|u|),
$$

where we have introduced for the probability density function (6.5) the notation

$$
h_{m}(x ;|u|)=\frac{H_{m}^{2}(x / \sqrt{2}|u|)}{m ! 2^{m}} \frac{e^{-x^{2} / 2|u|^{2}}}{\left(2 \pi|u|^{2}\right)^{1 / 2}} .
$$

In particular, the mean and the variance of the probability distribution (6.7) are given, respectively, by zero and

$$
\frac{|u|^{2}}{Z_{\beta, \lambda}} \sum_{m=0}^{\infty} \frac{e^{-\beta m}}{1-e^{-\beta(\lambda m+1)}} \frac{2 m+1}{m !} .
$$

Using the formulas (4.3) and (3.9), we find that the joint characteristic functions, in the given state, of the observables $A_{0}\left(u_{0}\right), A_{1}\left(u_{1}\right), \ldots, A_{k}\left(u_{k}\right)$ relative to the modes $0, \ldots, k$ are given by 


$$
\begin{aligned}
& \varphi\left[\exp \left[i \sum_{j=0}^{k} t_{j} A_{j}\left(u_{j}\right)\right]\right] \\
& \quad=\hat{p}_{A_{0}\left(u_{0}\right), A_{1}\left(u_{1}\right), \ldots, A_{k}\left(u_{k}\right)}\left(t_{0}, t_{1}, \ldots, t_{k} \mid \varphi\right) \\
& \quad=\sum_{m_{0}, m_{1}, \ldots, m_{k}} p_{m_{0}}^{0} p_{m_{0}, m_{1}} \cdots p_{m_{k-1}, k}\left\langle m_{0}, e^{i t_{0} A\left(u_{0}\right)} m_{0}\right\rangle\left\langle m_{1}, e^{i t_{1} A\left(u_{1}\right)} m_{1}\right\rangle \cdots\left\langle m_{k}, e^{i t A\left(u_{k}\right)} m_{k}\right\rangle \\
& \left.\quad=\left.\sum_{j_{0}, \ldots, j_{k}} p_{m_{0}}^{0} p_{m_{0}, m_{1}} \cdots p_{m_{k-1}, m_{k}} \frac{L_{m_{0}}\left(t_{0}^{2}\left|u_{0}\right|^{2}\right)}{m_{0} !} \frac{L_{m_{1}}\left(t_{1}^{2}\left|u_{1}\right|^{2}\right)}{m_{1} !} \cdots \frac{L_{m_{k}}\left(t_{k}^{2}\left|u_{k}\right|^{2}\right)}{m_{k} !} \exp \left|-\frac{1}{2} \sum_{j=0}^{k} t_{j}^{2}\right| u_{j}\right|^{2}\right) .
\end{aligned}
$$

Thus, in view of (4.1) and (4.2), the corresponding distributions are given by

$$
\begin{array}{r}
p_{A_{0}\left(u_{0}\right), \ldots, A_{k}\left(u_{k}\right)}\left(x_{0}, x_{1}, \ldots, x_{k} \mid \varphi\right)=\sum_{m_{0}, m_{1}, \ldots, m_{k}} \frac{1}{Z_{\beta, \lambda}} \prod_{j=1}^{k-1}\left(1-e^{-\beta\left(\lambda m_{j}+1\right)}\right) \exp \left[-\beta\left[\lambda \sum_{j=0}^{k-1} m_{j} m_{j+1}+\sum_{j=0}^{k} m_{j}\right]\right] \\
\times h_{m_{0}}\left(x_{0} ;\left|u_{0}\right|\right) h_{m_{1}}\left(x_{1} ;\left|u_{1}\right|\right) \cdots h_{m_{k}}\left(x_{k} ;\left|u_{k}\right|\right) .
\end{array}
$$

In particular if one chooses the parameters $u_{j} \in \mathbb{C}$ to be all equal to some nonzero complex number $u$, then one obtains a process $\left[A_{j}(u)\right]$ which is translation invariant but not Markovian.

Using the expression (3.11) for the transition operators $P_{j}$ and the explicit form (2.24) for the Heisenberg dynamics, one can easily compute the correlation functions of an observable $A_{j}$ relative to the $j$ th mode of the field. If $j \geq 1$ one finds

$$
\left\langle A_{j} A_{j}(t)\right\rangle=\operatorname{Tr}\left(w_{0} P_{0}^{1-i t} A_{1} P_{0}^{i t} P_{1}^{i t} A_{1} P_{1}^{1-i t}\right),
$$

where $\operatorname{Tr}$ means that one takes the trace over all the Hilbert spaces involved. Using the expression (3.12) for the quantum transition operator and the simplifying notation (3.13), the right-hand side of (6.12) can be written as the trace of

$$
\sum_{\substack{n_{0}, m_{1}, n_{1}}}\left|n_{0}\right\rangle\left\langle n_{0}\left|\otimes \frac{e^{-(1-i t) \beta\left(\lambda n_{0}+1\right) N_{1}}}{Z^{(1-i t)}\left(n_{0}\right)} A_{1} \frac{e^{-i t \beta\left(\lambda n_{0}+1\right) N_{1}}}{Z^{i t}\left(n_{0}\right)}\right| n_{1}\right\rangle\left\langle n_{1}\left|A_{1}\right| m_{1}\right\rangle\left\langle m_{1}\right| \otimes \frac{e^{-i t \beta\left(\lambda n_{1}+1\right) N_{2}-(1-i t) \beta\left(\lambda m_{1}+1\right) N_{2}}}{Z^{i t}\left(n_{1}\right) Z^{(1-i t)}\left(m_{1}\right)} .
$$

Taking the trace (over $\left.\mathscr{H}_{0} \otimes \mathscr{H}_{1} \otimes \mathscr{H}_{2}\right)$ of this expression and recalling that $Z\left(n_{0}\right)=\left(1-e^{-\beta\left(\lambda n_{0}+1\right)}\right)^{-1}$, one finds

$$
\sum_{\substack{m_{0}, n_{2} \\ m_{1}, n_{1}}} \frac{\left|\left\langle m_{1}, A n_{1}\right\rangle\right|^{2}}{Z_{\beta, \lambda} Z^{i t}\left(n_{1}\right) Z^{1-i t}\left(m_{1}\right)} e^{-\beta n_{0}-i t \beta\left(\lambda n_{0}+1\right) n_{1}} e^{-(1-i t) \beta\left(\lambda n_{0}+1\right) m_{1}} e^{-\beta\left[\left(\lambda m_{1}+1\right)+i t \lambda\left(n_{1}-m_{1}\right)\right] n_{2}} .
$$

Hence, summing over $n_{0}, n_{2}$ one arrives at the result:

$$
\left\langle A_{j} A_{j}(t)\right\rangle=\sum_{m, n} \frac{\left\langle\left.\langle m, A n\rangle\right|^{2}\right.}{Z_{\beta, \lambda}}\left[\frac{1-e^{-\beta(\lambda n+1)}}{1-e^{-\beta(\lambda m+1)}}\right]^{i t} \frac{\left(1-e^{-\beta(\lambda m+1)}\right) e^{-\beta[m+i t(n-m)]}}{\left(1-e^{-\beta[1+\lambda m+i t \lambda(n-m)]}\right)^{2}} .
$$

A similar calculation for the zero mode yields the result

$$
\left\langle A_{0} A_{0}(t)\right\rangle=\operatorname{Tr}\left[P_{1}\left(w_{0} P_{0}\right)^{1-i t} A_{0}\left(w_{0} P_{0}\right)^{i t} A_{0}\right]=\sum_{m, n} \frac{|\langle m, A n\rangle|^{2}}{Z_{\beta, \lambda}} \frac{e^{-\beta[m+i t(n-m)]}}{1-e^{-\beta[1+\lambda m+\lambda i t(n-m)]}} .
$$

\section{ACKNOWLEDGMENTS}

This paper was written with the support of Grant No. MCS 821381 from the National Science Foundation and Contract No. N00014-84-K-0421 from the Office of Naval Research to Princeton University.

${ }^{1}$ L. Accardi, Phys. Rep. 77, 169 (1981).

${ }^{2}$ R. Courant and D. Hilbert, Methods of Mathematical Physics (Interscience, New York, 1953).

${ }^{3}$ V. K. Fedanin, V. G. Makhankov, and L. V. Yakushevich, Phys. Lett. 614, 256 (1977).
${ }^{4}$ I. S. Gradshteyn and I. M. Ryzhik, Table of Integrals, Series and Products (Academic, Orlando, 1980).

${ }^{5}$ M. Rosenblatt, Markov Processes. Structure and Asymptotic Behavior (Springer, Berlin, 1971). 